\title{
Coating Film Profiles Generated by Fluctuating Location of the Wiping Pressure and Shear Stress
}

\author{
Andrew Dennis JOHNSTONE, ${ }^{1)}$ Buyung KOSASIH, ${ }^{1) *}$ Le Quang PHAN, ${ }^{1)}$ Andrew DIXON ${ }^{2)}$ and \\ Wayne RENSHAW ${ }^{3)}$
}

1) ARC Research Hub for Australian Steel Manufacturing, School of Mechanical, Materials, Mechatronic and Biomedical Engineering, University of Wollongong, Northfields Avenue, NSW 2522 Australia.

2) ARC Research Hub for Australian Steel Manufacturing, Faculty of Engineering and Information Sciences, University of Wollongong, Northfields Avenue, NSW 2522 Australia.

3) Bluescope Steel Pty Ltd, Port Kembla, NSW 2505 Australia.

(Received on June 13, 2018; accepted on October 11, 2018; J-STAGE Advance published date: December 1, 2018)

\begin{abstract}
Impinging planar jets are a widely used means of removing excess drag-out coating material from steel strip in order to control the final thickness of the applied coating. A wide range of possible coating defects are known to occur for this process, many of which are suspected to have their origin in the spatiotemporal characteristics of the air jets. It is therefore of interest to improve understanding of the link between the unsteady flow behaviour inherent to impinging jets and the evolution of the coating free surface produced by the gas-wiping process. In this paper, the coating response, characterised by the amplitude and frequency of the coating thickness fluctuation, throughout both the active region of the gas-wiping jets and the region immediately downstream, is investigated using a numerical model. The pressure and shear stress profiles acting on the coating surface along the strip are imposed as timevarying inputs such that for both the pressure and shear the vertical location of the entire profile undergoes sinusoidal oscillation parallel to the strip. A range of amplitude-frequency combinations for the vertical oscillation of the profiles are employed to assess the combined effect of these parameters on the coating response. Additionally, the strip speed is a varied parameter. Both the magnitude of the coating thickness fluctuation and the corresponding shape of the coating surface profile along the strip are found to be dependent on the strip speed and the oscillation amplitude and frequency of the vertical location of the pressure and shear stress profiles.
\end{abstract}

KEY WORDS: gas-wiping; coating; Navier-Stokes equations; thin film; viscous flow.

\section{Introduction}

In industrial continuous galvanising lines (CGL) impinging gas jets are often employed to remove excess coating material from the steel strip. The majority of the coating fluid dragged up from the molten metallic alloy bath is removed by this process-and returned to the bath-leaving a very thin film of the metallic alloy coating to remain on the strip. As the strip exits upwards out of the bath the drag-out thickness of the coating is of mm-order and after the strip passes through the active region of jet-impingement the coating thickness is reduced to $\mu$ m-order.

Achieving a target average final (i.e. post-jet impingement region) coating thickness requires an appropriate combination of the set of control parameters of the process: the strip speed $U$, mean velocity of the gas exiting the jet lip gap $U_{o}$ (i.e. jet plenum pressure) the offset of the jet opening from the strip $H$, the size of the jet lip gap $d$ and the angle the jet centreline makes with the horizontal $\theta$. These settings

\footnotetext{
* Corresponding author: E-mail: buyung@uow.edu.au DOI: https://doi.org/10.2355/isijinternational.ISIJINT-2018-413
}

for the control parameters inherently account for the values of the coating properties (e.g. density $\rho$, viscosity $\mu$ and surface tension $\sigma$ ) that apply to the alloying material when in the liquid state at a temperature reasonably assumed to be equal to the bath temperature. The basis for this temperature assumption is the combination of the close proximity of the jet-wiping point to the bath and the relatively high speed of the strip in a typical industrial process configuration which accords to short travel time $(\sim 0.2 \mathrm{~s})$ of the strip between the bath surface and the location of the wiping jets.

The surface of the final coating produced by the jet-wiping process on continuous galvanising lines typically is characterised by waviness and other undesirable features, all of which detract from the quality of the final product. In more extreme cases, coating surface defects can lead to product rejection and therefore material and energy wastage. Even in the absence of serious defects, 'good quality' surface coatings of acceptable production standard produced using CGL still have present a measurable degree of roughness.

Ideally, in the CGL process the excess drag-out coating material would be removed by a perfectly steady wiping 'tool'. However, this is not the nature of the wiping 'tool' 
when using gas jets as the means of coating removal. Free jet flows are well known to exhibit fluctuations ${ }^{1-7)}$ which occur over a broad frequency range and for the case of jets impinging on a surface this inherent unsteadiness remains a primary feature of the jet flow. ${ }^{8-12)}$ One characteristic of the impinging jet flow is the jet buckling mode observed in the case of asymmetric jet flows. ${ }^{9)}$ The jet buckling is directly associated with the presence and instantaneous locations of the large-scale vortex structures which evolve along the shear boundaries between the jet and the surrounding air due to the velocity gradients that exist across these boundaries, particularly over the early part of the jet flow. These convecting large-scale vortex structures are localised regions of low relative pressure and consequently are a primary cause of the jet buckling wherein the jet flow is deflected locally outward toward the respective vortex positions. Higher buckling modes have a correspondingly higher number of large-scale vortices distributed between the jet exit and the impingement surface.

Owing to the nature of their effect on the behaviour of the impinging jet flow the large-scale vortex structures are a principal reason for the fluctuation in the action of the jet on an impingement surface. As a consequence, temporal variation of the pressure at the impingement surface $^{13,14)}$ unavoidably occurs. Additionally, the spatiotemporal behaviour of the large-scale recirculating eddies located immediately outside the impingement zone of the jet are an additional likely cause of jet instability. ${ }^{11,15,16)}$ Owing to these aforementioned behavioural characteristics of the impinging jet flow, jet driven inconsistency in the thickness of the coating-and, concomitantly, the surface quality-produced on CGL may be anticipated, particularly at operating points (settings) at which the coating may be highly sensitive to perturbations at the frequencies inherent to the impinging jet.

In developing the analytic model we have sourced an approximate mathematical model for the thin viscous coating in the jet-wiping problem from the literature ${ }^{17)}$ and employed a numerical scheme to obtain the solution for the free-surface of the coating along the strip for the case of temporal variation in the vertical location of the pressure and shear stress acting on the coating. In the simulation cases discussed in this report the along-strip location of the pressure and shear stress profiles oscillate with a temporal sinusoidal pattern. This approach was adopted, at this stage in our research, in order to mimic in a straightforward way the flapping mode of impinging jet instability-a mode identified as one of the principle modes of jet instability associated with the coherent structures of the jet and determined to be present in significant strength across a broad range of frequencies, ${ }^{12)}$ including low frequencies that compare well to the frequency of coating thickness fluctuations obtained numerically ${ }^{12)}$ and as observed on CGL. The amplitude $a_{p o s}$ and frequency $f_{p o s}$ of the vertical oscillation of the pressure and shear stress profiles were varied between different simulation runs in order to assess their combined effect on the coating response; additionally, strip speed $U$ was a varied parameter. Consequently, the present simulation results are quantitative predictions for the amplitude of the undulations produced along the coating surface under conditions of the coating being subjected to different discrete perturbation fre- quencies of the wiping actuators i.e. the pressure and shear stress profiles of the wiping jet. This approach is comparable to generating response-amplitude-operator (RAO) maps for dynamic systems. It will be seen that the magnitude of the coating thickness fluctuation relative to the mean thickness and, furthermore, the shape of the coating surface profile along the strip are found to be dependent on the nondimensional frequency $d f_{\text {pos }} / U$, where $d$ is the jet lip gap.

At this stage in the development of the numerical model reported herein, inertia terms and surface tension are omitted. Thus, the coating is treated as a lubrication-film model with zero surface tension. Inertia is, however, known to have a destabilising effect on films of the type considered herein. ${ }^{18)}$ It may therefore be anticipated that the modelling of the coating response with inertia neglected will return conservative results $e . g$. the threshold for instability and the magnitude of variation in the coating thickness may be overand underestimated, respectively, by such an approach.

\section{Mathematical Model of Coating Thickness}

The mathematical model that forms the basis of the present work is as formulated in the earlier work of other authors. ${ }^{17,19,20)}$ Overall, the general conditions of the model are that the coating is mathematically treated using the thin film assumptions and is assumed to be two-dimensional. Following the application of the usual boundary conditions for the jet wiping problem to the equations governing the flow, a PDE that specifies the coating surface $h$ as a function of strip location $x$ and time $t$ can be formulated, and we have sourced such an equation from an earlier study ${ }^{17)}$ for use as the basis of the model reported herein, specifically (with the surface tension term omitted),

$$
\hat{h}_{\hat{t}}+Q_{\hat{x}}=0
$$

using,

$$
\begin{gathered}
\hat{h}=h /[\mu U /(\rho g)]^{1 / 2} \\
\hat{t}=(U / d) t, \text { and } \ldots . . \\
\hat{x}=x / d, \ldots \ldots \ldots . . .
\end{gathered}
$$

where, $\mu$ and $\rho$ are, respectively, the dynamic viscosity and density of the liquid coating, with values in the present work taken to be $\mu=0.0010$ Pa.s and $\rho=3000 \mathrm{~kg} / \mathrm{m}^{3}$.

In addition,

$$
Q=\left(\hat{h}+\frac{1}{2} \hat{h}^{2} \mathrm{G}(\hat{x})-\frac{1}{3} \hat{h}^{3}\left(\mathrm{~S}+\mathrm{P}^{\prime}(\hat{x})\right) .\right.
$$

where, $Q$ represents the local volume flow flux and $\mathrm{S}$ is the nondimensional Stokes number. $\mathrm{G}(\hat{x})$ and $\mathrm{P}(\hat{x})$ are nondimensional pressure and shear acting on the coating surface, where $\mathrm{G}(\hat{x})=\tau(\hat{x}) \varepsilon L /(\mu U)$ and $\mathrm{P}(\hat{x})=p(\hat{x}) \varepsilon^{2} L /(\mu U)$. For convenience, from this point forward the symbol ' ${ }^{\wedge}$ ' is omitted from the notation in Eqs. (4)-(13) whilst the same meaning is implied throughout.

To obtain the steady state solutions in the present work (which subsequently served as the starting conditions for the 
unsteady simulations) we follow the process developed previously ${ }^{17)}$ for finding the thickness profile from far upstream to far downstream such that the volume flux is constant and corresponds to that necessary for the desired downstream coating thickness. It can be shown that

$$
Q_{x}=h_{x}\left\{1+h \mathrm{G}(x)-h^{2}\left(\mathrm{~S}+\mathrm{P}^{\prime}(x)\right)\right\}+\frac{1}{2} h^{2} \mathrm{G}^{\prime}(x)-\frac{1}{3} h^{3} \mathrm{P}^{\prime \prime}(x)
$$

and hence Eq. (1) can be rewritten as

$$
h_{t}+C h_{x}=A
$$

with

$$
C=1+h_{c} \mathrm{G}\left(x_{c}\right)-h_{c}^{2}\left(\mathrm{~S}+\mathrm{P}^{\prime}\left(x_{c}\right)\right)
$$

and

$$
A=\frac{1}{3} h^{3} \mathrm{P}^{\prime \prime}(x, t)-\frac{1}{2} h^{2} \mathrm{G}^{\prime}(x, t) .
$$

It is interesting to note that for $C$ varying slowly then the non-steady solutions to Eq. (5) can be interpreted as a wave travelling at speed $C$ being forced by the term $A$. Under different conditions it is possible for this forcing to either attenuate or increase the wave amplitude.

\section{Methodology}

For obtaining the steady-state solutions, the pressure and shear stress profiles, respectively, that form the basis of the terms $\mathrm{P}(x)$ and $\mathrm{G}(x)$ employed in Eqs. (3), (4) \& (5) are:

$$
\begin{gathered}
p(x)=p_{\max }\left\{\exp \left(-(0.9 x)^{2}\right)\right\} \ldots \ldots \ldots . . \\
\tau(x)=\tau_{\max }(x) /\left\{1+(0.7 x \times \operatorname{sign}(x))^{1.9}\right\}
\end{gathered}
$$

In all cases of the present work the profiles (Eqs. (6) and (7)) and peak magnitudes of the pressure and shear accord closely to those reported in the literature corresponding to a jet Reynolds number of $R e \sim 7 \times 10^{3}$ and jet-to-surface offset of $H / d \sim 8 .^{21)}$ Examples of the profiles given by Eqs. (6) and (7) are shown in Fig. 1 for $p_{\max }=5 \mathrm{kPa}$ and $\tau_{\max }=50$ $\mathrm{Pa}$. The majority of simulations were conducted within the range of strip locations $-2 \leq \hat{x} \leq 8$.

The ranges across which the parameters were varied in the simulations reported herein are presented in Table 1. With the exception of $U=3 \mathrm{~m} / \mathrm{s}$, for each strip speed many combinations of oscillation amplitude $a_{\text {pos }} / d$ and frequency $f_{\text {pos }}$ of the pressure and shear stress profiles were employed.

As stated, the steady-state solutions obtained using Eqs. (1), (3)-(7) served as the starting profiles of the coating surface for the simulations that included time-varying magnitude of the pressure and shear stress. At each timestep $i$ in the unsteady solution the procedure consisted of solving Eq. (5) by:

1. Calculating the expressions for the speed of disturbance propagation $C$ and the growth (or decay) $A$ at each $x$ location, using Eqs. (8) and (9), respectively,

$$
\begin{gathered}
C_{x, i}=1+h_{x, i} \mathrm{G}(x, t)-h_{x, i}^{2}\left(\mathrm{~S}+\mathrm{P}^{\prime}(x, t)\right) \\
A_{x, i}=\frac{1}{3} h_{x, i}^{3} \mathrm{P}^{\prime \prime}(x, t)-\frac{1}{2} h_{x, i}^{2} \mathrm{G}^{\prime}(x, t) \ldots
\end{gathered}
$$

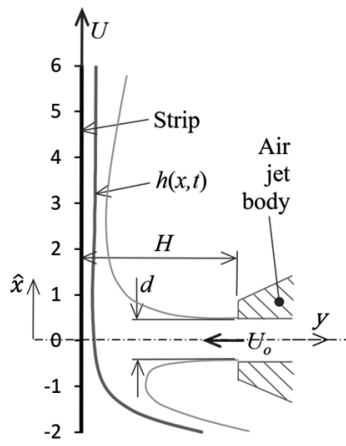

(a)

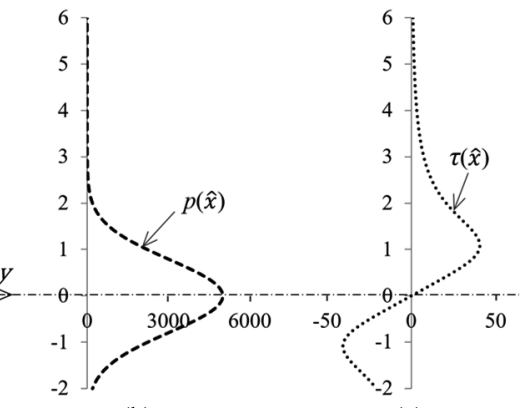

(b)

(c)
Fig. 1. a) Schematic of jet wiping process (coating thickness and time-averaged location of the jet flow boundaries not to scale); b) Pressure acting on coating surface (Pa); and c) Shear stress acting on coating surface $(\mathrm{Pa})$.

Table 1. Range of parameter values tested in the simulations.

\begin{tabular}{cccc}
\hline Strip speed, $U[\mathrm{~m} / \mathrm{s}]$ & $a_{\text {pos }} / d$ & $f_{\text {pos }}[\mathrm{Hz}]$ & $d f_{\text {pos }} / U$ \\
\hline 1.00 & $0.0318-1.25$ & $30.0-1000$ & $0.0300-1.00$ \\
1.15 & $0.420-0.580$ & $173-805$ & $0.150-0.700$ \\
1.50 & $0.350-1.17$ & $188-1250$ & $0.125-0.833$ \\
2.00 & $0.150-1.18$ & $200-4000$ & $0.100-2.00$ \\
3.00 & 0.500 & $180 \& 3000$ & $0.0600 \& 1.00$ \\
\hline
\end{tabular}

2. Calculating the new coating thickness $h$ at timestep $i+$ 1 for each $x$ location using Eq. (10).

$$
h_{x, i+1}=h_{x, i}+\Delta t\left\{A_{x, i}-C_{x, i}\left(h_{x+1, i}-h_{x-1, i}\right) /(2 \Delta x)+M_{x, i}\right\}
$$

where,

$$
M_{x, i}=\left(C_{x, i} / \Delta x\right)^{2}(\Delta t / 2)\left(h_{x+1, i}-2 h_{x, i}+h_{x-1, i}\right) .
$$

Due to the imposed oscillation in the vertical location of the pressure and shear stress profiles Eqs. (8) and (9) are functions of space and time. The expressions for the terms $\mathrm{P}(x, t)$ and $\mathrm{G}(x, t)$ are:

$$
\begin{aligned}
& \mathrm{G}(x, t)=\mathrm{G}(X) \\
& \mathrm{P}(x, t)=\mathrm{P}(X) .
\end{aligned}
$$

where,

$$
X=x+\left(a_{\text {pos }} / d\right) \sin \left(2 \pi f_{\text {pos }} t\right)
$$

\section{Simulation Results and Discussion}

Throughout, it will be seen that when the coating amplitude response data of the various strip velocities of the simulations (i.e. $U=1.00,1.15,1.50,2.00 \& 3.00 \mathrm{~m} / \mathrm{s}$ ) is expressed using the $r_{\text {pos }}$ parameter the data collapses to a single curve when plotted against nondimensional frequency $d f_{\text {pos }} / U$.

$$
r_{p o s}=\frac{\hat{h}_{r m s} / \hat{h}_{\text {mean }}}{2 \pi a_{\text {pos }} f_{\text {pos }} / U}
$$

where, 


$$
\hat{h}_{\mathrm{rms}}=\left[\left\{\sum_{i=1}^{n}\left(\hat{h}_{i}-\hat{h}_{\text {mean }}\right)^{2}\right\} /(n+1)\right]^{1 / 2},
$$

$\hat{h}_{\text {mean }}$ is the average (nondimensional) thickness of the coating, calculated using the time traces of the coating thickness at a given $\hat{x}$ coordinate (e.g. at $\hat{x}=5)$ over the full simulation time, and $n$ is the number of time-steps throughout a simulation.

Note that in a physical sense $d f_{\text {pos }} / U$ represents the ratio of the time taken for a fixed point on the strip to travel a distance equal to the size of the jet lip-gap (time for strip to travel $1 d=d / U$ ) to the duration of a single cycle of the jet pressure and shear stress oscillation (duration of oscillation cycle $\left.=1 / f_{p o s}\right)$. It will be seen that there is a particular value of $d f_{p o s} / U$ at which the response amplitude of the coating surface is most sensitive to the perturbations of the wiping actuators.

Before moving on to the overall amplitude response results of the coating for the full set of simulations undertaken (i.e. Fig. 6), the surface profile results for three different cases representative of those corresponding to operating points in the simulations of low, intermediate and high nondimensional frequency are presented, respectively, in Figs. $2,3 \& 4$; with each figure showing the surface profile generated throughout one full oscillation cycle of the pressure and shear stress profiles.

There is apparent a general overall trend of reduction in both the response wavelength and-with reference to Fig. 6-the amplitude (relative to the average thickness) of the coating thickness fluctuation as the oscillation frequency of the pressure and shear is increased. At nondimensional forcing frequencies in the range $d f_{\text {pos }} / U>1$ (the 'slow' strip-'fast' jet oscillation condition) the fluctuations in the coating thickness are small (see Figs. 4, 6, $7 \&$ 9(c)). Conversely, when the period of lip-gap crossing of the strip is

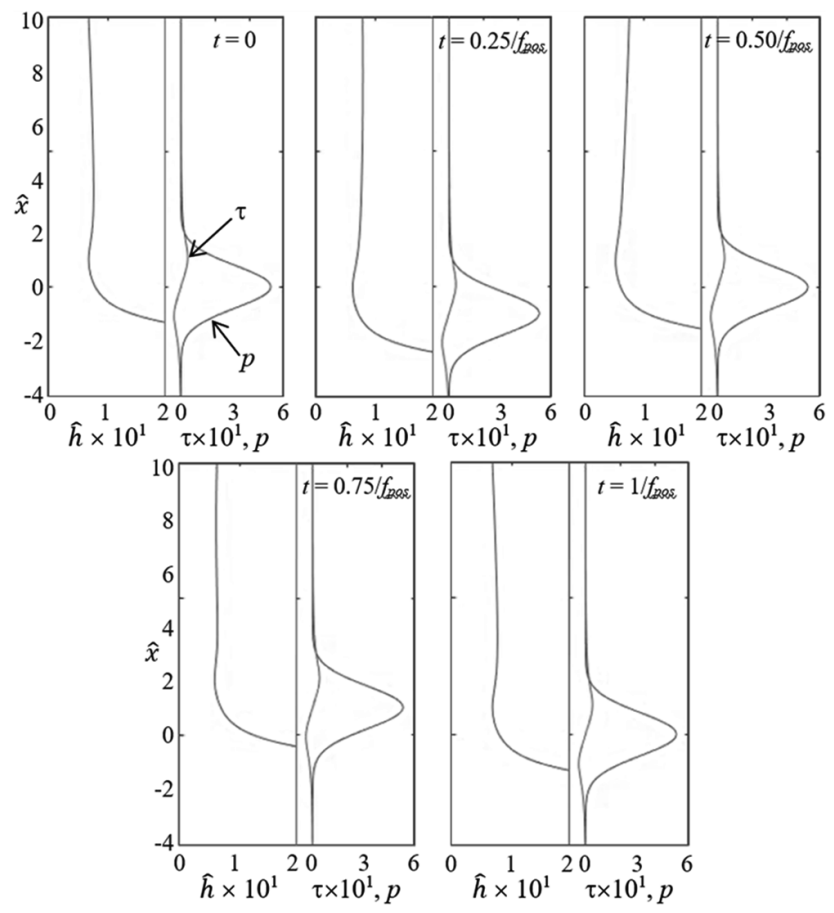

Fig. 2. Case 1: Coating surface profiles over one oscillation cycle of pressure $p(\mathrm{kPa})$ and shear $\tau(\mathrm{kPa})$ at low nondimensional frequency, $d f_{\text {pos }} / U=0.030 ; U=1.0 \mathrm{~m} / \mathrm{s}, a_{p o s} / d=$ $1.0, f_{\text {pos }}=30 \mathrm{~Hz}$. less than approximately $10 \%$ of the period of jet oscillations (i.e. at $d f_{\text {pos }} / U<\sim 0.1$; the 'fast' strip-'slow' jet oscillation condition) the variation in the coating thickness is approximately two orders of magnitude greater (see Figs. 2, 6, 7 \& 9 (a)) and, correspondingly, the wavelength $\lambda$ of the coating surface is longer (see Fig. 6). In Fig. 5 a series of coating profiles, this time for the strip speed held constant at $U=$ $3 \mathrm{~m} / \mathrm{s}$, over the range of frequencies $f_{\text {pos }}=180,330,900$, 1800,2400 and $3000 \mathrm{~Hz}$ is presented in which the dependence of the coating thickness fluctuation on the oscillation

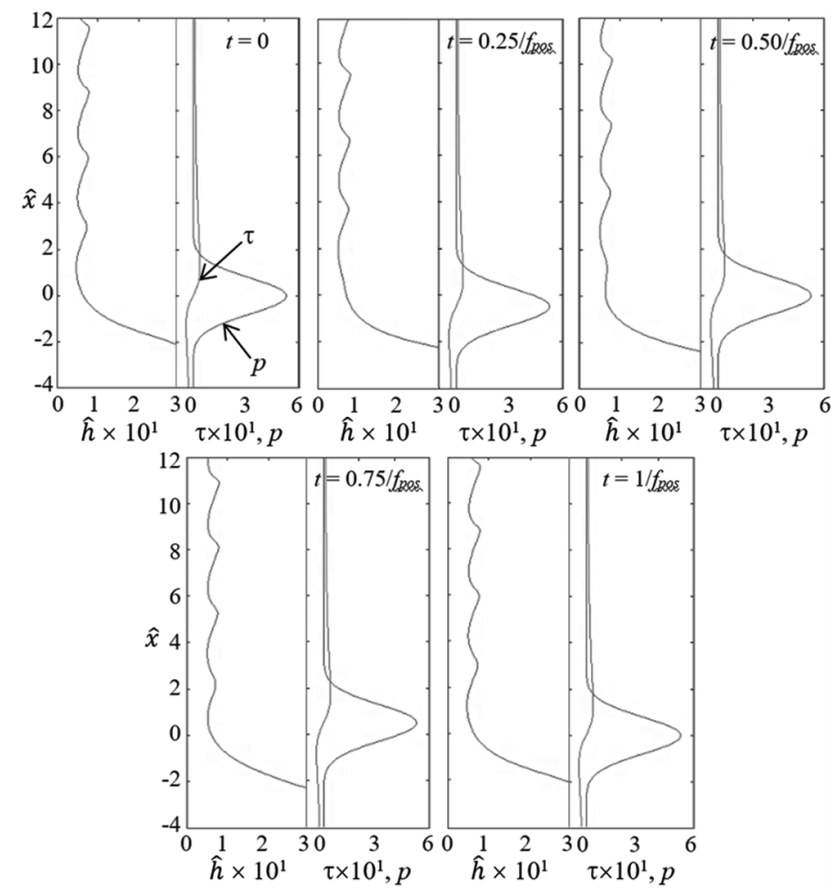

Fig. 3. Case 2: Coating surface profiles over one oscillation cycle of pressure $p(\mathrm{kPa})$ and shear $\tau(\mathrm{kPa})$ at intermediate nondimensional frequency, $d f_{p o s} / U=0.40 ; U=1.5 \mathrm{~m} / \mathrm{s}$, $a_{p o s} / d=0.5, f_{\text {pos }}=600 \mathrm{~Hz}$.

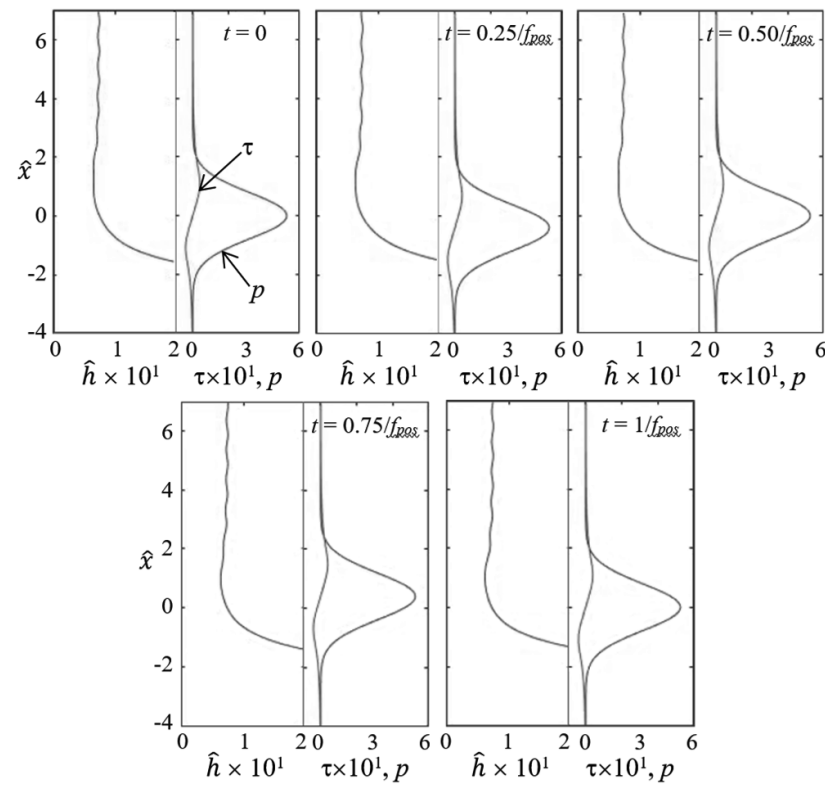

Fig. 4. Case 3: Coating surface profiles over one oscillation cycle of pressure $p(\mathrm{kPa})$ and shear $\tau(\mathrm{kPa})$ at high nondimensional frequency, $d f_{p o s} / U=1.0 ; U=2.0 \mathrm{~m} / \mathrm{s}, a_{p o s} / d=0.4$, $f_{\text {pos }}=2000 \mathrm{~Hz}$. 


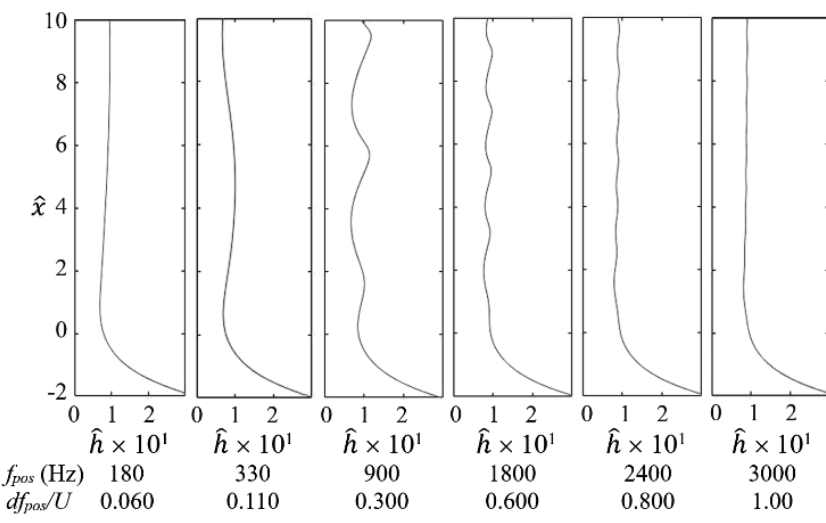

Fig. 5. Coating surface profiles at various oscillation frequencies $f_{p o s}$ of the pressure and shear stress for the case of strip speed $U=3 \mathrm{~m} / \mathrm{s}$ with $a_{p o s} / d=0.5$.

Table 2. The three coating response modes and approximate $d f_{\text {pos }} / U$ boundaries.

\begin{tabular}{ccc}
\hline $\begin{array}{c}\text { Long ripple-large } \\
\text { amplitude }\end{array}$ & $\begin{array}{c}\text { Asymmetric-sharp } \\
\text { crest }\end{array}$ & $\begin{array}{c}\text { Short ripple- } \\
\text { small amplitude }\end{array}$ \\
\hline$d f_{p o s} / U \leq 1 / 5$ & $1 / 5<d f_{p o s} / U<1 / 2$ & $d f_{p o s} / U \geq 1 / 2$
\end{tabular}

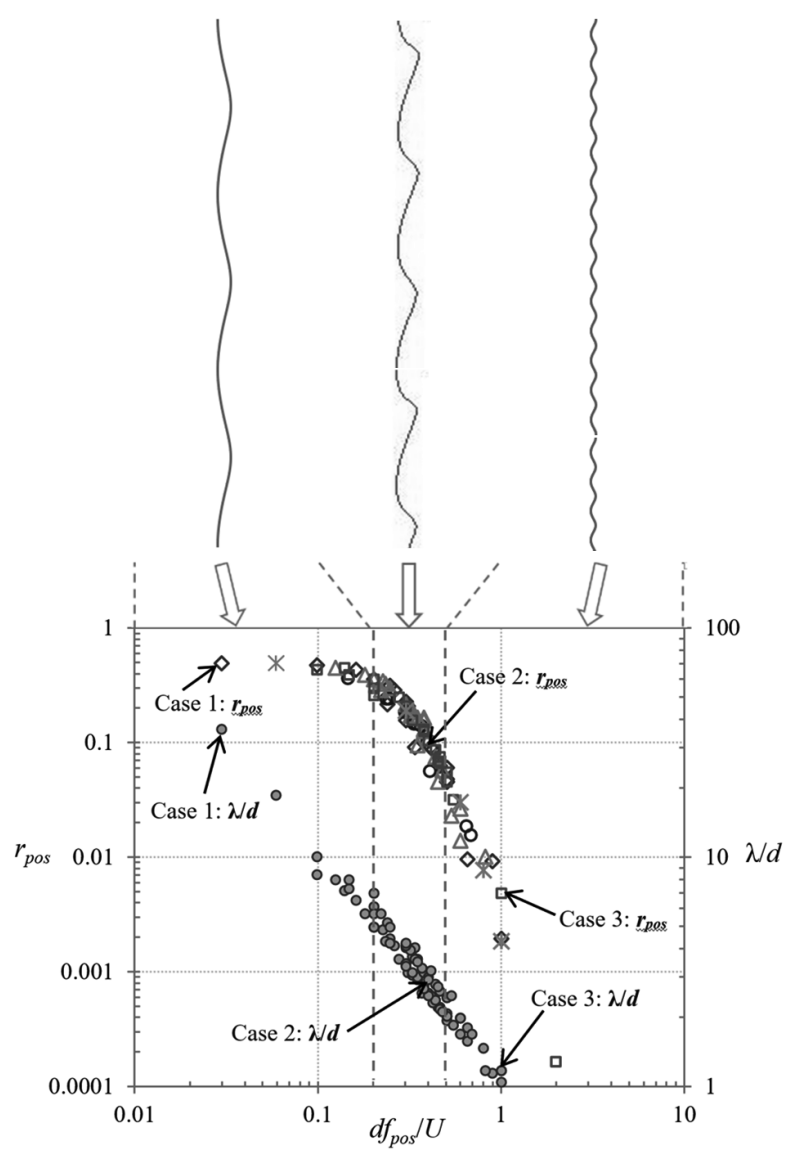

$\diamond \mathrm{U}=1.0 \mathrm{~m} / \mathrm{s} \quad \circ \mathrm{U}=1.15 \mathrm{~m} / \mathrm{s} \quad \Delta \mathrm{U}=1.5 \mathrm{~m} / \mathrm{s} \quad \square \mathrm{U}=2.0 \mathrm{~m} / \mathrm{s} \quad \nVdash \mathrm{U}=3.0 \mathrm{~m} / \mathrm{s} \quad$ o Surface wavelength, all $\mathrm{U}$

Fig. 6. Coating amplitude response parameter, $r_{p o s}$ (values at $\hat{x}=$ 5 ) and nondimensional wavelength $\lambda / d$ of the coating surface profile.

frequency of the pressure and shear is further observable.

Overall, these general trends are identical to those seen in the preceding paper for oscillation in the magnitude of the pressure and shear stress profiles with the centre of the profiles fixed at $\hat{x}=0 .{ }^{22}$ However, unlike the previous

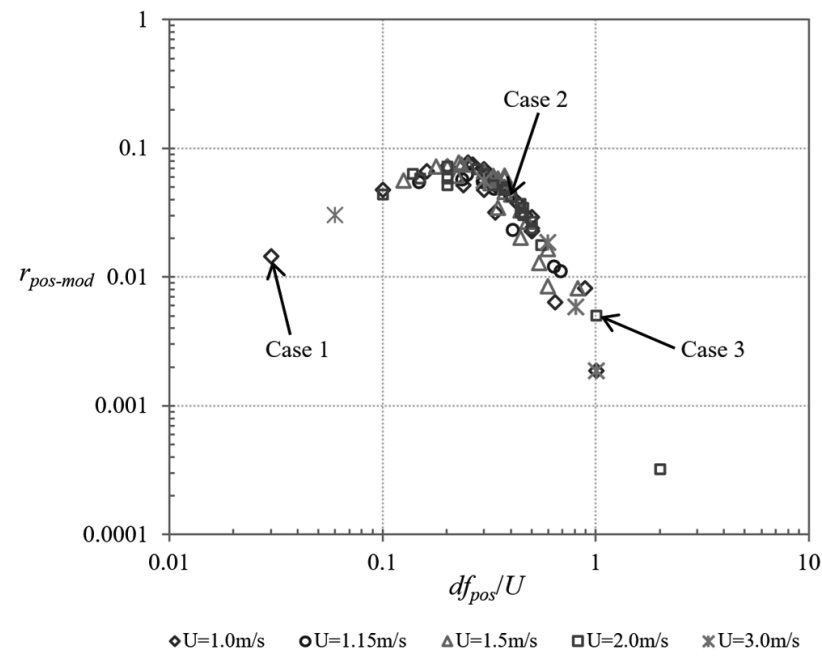

Fig. 7. Modified coating amplitude response parameter, $r_{p o s-m o d}$ (values at $\hat{x}=5$ ).

results $^{22)}$ the outcomes of the present work for vertical oscillation in the location of the pressure and shear stress profiles show a special case to exist for the shape of the surface profile for operating points at intermediate nondimensional frequencies i.e. intermediate $d f_{\text {pos }} / U$. This outcome is discussed in detail in the sections that follow.

The pattern of repetitious variation in the thickness of the coating along the strip in the present simulations (see the example cases in Figs. 2-4) is fundamentally a consequence of the motion of the pressure and shear stress profiles relative to the motion of the strip. During each oscillation cycle of the location of the pressure and shear stress profiles, the interval of upwards motion of the profiles is effectively a state of slower strip for static profiles, and thus a thinner coating results. Conversely, and for the same reason, during the interval of downwards motion of the pressure and shear stress profiles (effectively a state of faster strip for static profiles) a thicker coating results. For these reasons the amplitude-given its link to velocity-of the pressure and shear stress oscillations (i.e. how far the limits of their vertical motion are from the nominal centreline) is a key factor on which the degree of variation in the coating thickness over each ripple length is dependent.

In regard to the shape of the surface profile at both small and large $d f_{\text {pos }} / U$ (e.g. Case 1 and Case 3, respectively) the profile can be characterised as highly sinusoidal. However, this does not apply to scenarios at nondimensional frequencies within the approximate range $1 / 5<d f_{\text {pos }} / U<1 / 2$ (e.g. Case 2). Simulation results at these intermediate nondimensional frequencies are special cases exhibiting a surface profile characterised by sharper, narrower crests and more drawn out troughs.

Considering further the simulation results for nondimensional frequencies within the intermediate range $1 / 5<$ $d f_{\text {pos }} / U<1 / 2$, after the initial development of the surface profile the nature of the surface is one of a high degree of symmetry about the central point of the surface crest (see e.g. Fig. 3 and the coating surface profile in the region $1<\hat{x}<3$ at $\left.t=0.75 / f_{\text {pos }}\right)$. As the coating is then carried upward with the movement of the strip the location of this initial crest migrates upwards and, consequently, out of the area of influence of the pressure profile (or more precisely, 
the pressure gradient) acting on the coating. However, the influence of the shear stress acting on the surface of the coating extends, for the profiles in the present simulations, at significant levels up to around $\hat{x}=8$.

Under the laminar, thin film conditions of the present problem the coating is satisfactorily assumed to possess a linear velocity profile in response to shear stress imposed on the coating in the direction parallel to the strip. Under this fluid-dynamic condition the surface of a thicker (thin) film (in the present case, the crest region) has to travel faster relative to the substrate (strip) than a thinner film (in the present case, the trough region) in order to generate the stress response of equal magnitude to a given shear stress imposed at the coating surface; that is, to generate the same strain rate in the fluid. This is the reason for the asymmetry that develops in the crests (most of which occurs within the region $0<\hat{x}<4$ throughout which the shear stress acting on the coating is still of significant magnitude) as they are carried upwards, the effect of which is amplified by the monotonic reduction in the magnitude of the shear stress imposed on the coating away from the impingement region of the jet i.e. the surface shear stress at a trough is slightly less than that acting on the crest located immediately below. For example, at $t=$ $0.75 / f_{\text {pos }}$ in Fig. 3 it is noteworthy to compare the skewed crest at $\hat{x}=8$ against the highly symmetric crest at $\hat{x}=2$. Overall, this outcome indicates that for the present simulation scenario (i.e. the vertical location of the entire pressure and shear stress profiles undergoing oscillation parallel to the strip) at nondimensional frequencies within the approximate range $1 / 5<d f_{\text {pos }} / U<1 / 2$ the shear stress acting on the coating is playing a significant role in the development of the shape of the surface profile throughout the region $0<\hat{x}<\sim 8$.

Additional insight can be gained regarding the response behaviour of the coating to unsteady pressure and shear stress profiles acting on the coating by plotting the data using the modified amplitude response parameter, given by

$$
r_{p o s-m o d}=\frac{\hat{h}_{r m s} / \hat{h}_{\text {mean }}}{2 \pi a_{\text {pos }} / d}
$$

The modified parameter $r_{\text {pos-mod }}$ is particularly useful for highlighting the frequency dependence of the amplitude response of the coating (i.e. the amplitude of the coating ripple generated by oscillation in the vertical location of the pressure and shear stress profiles). As Fig. 7 shows, the largest ripple amplitudes (relative to the average thickness of the coating) occur roughly within the intermediate range of nondimensional frequency, with the peak occurring at $d f_{\text {pos }} / U \sim 0.22$; at this particular operating point the strip travels a distance of almost 5 times the jet lip gap (i.e. a distance of $\sim 5 d$ ) during each oscillation cycle of the pressure and shear stress profiles.

A series of curves can be generated from the data of Fig. 7 to highlight the role strip speed plays in the sensitivity of the coating to wiping perturbations. Figure 8 presents a family of $r_{\text {pos-mod }}$ curves for four different strip speeds $U=$ $1,2,3 \& 4 \mathrm{~m} / \mathrm{s}$ for a jet lip gap of $d=1 \mathrm{~mm}$.

It can be seen in Fig. 8 that at higher strip speeds the bandwidth of the frequency sensitivity of the coating is increased. Furthermore, the increase in bandwidth is directly proportional to the increase in the strip speed. For example,

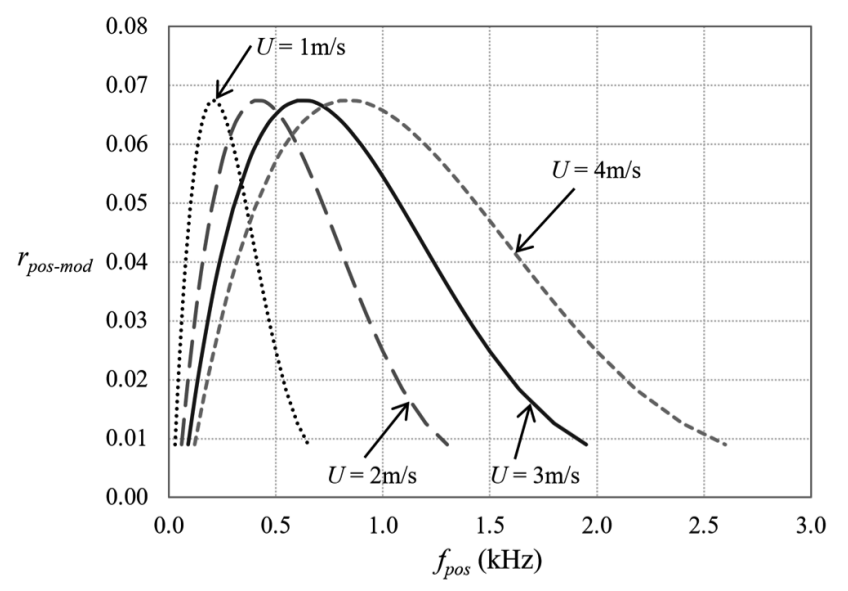

Fig. 8. Role of strip speed in coating sensitivity to wiping perturbations (curves for $\hat{x}=5$ ). Curves shown correspond to jet lip gap of $d=1 \mathrm{~mm}$.

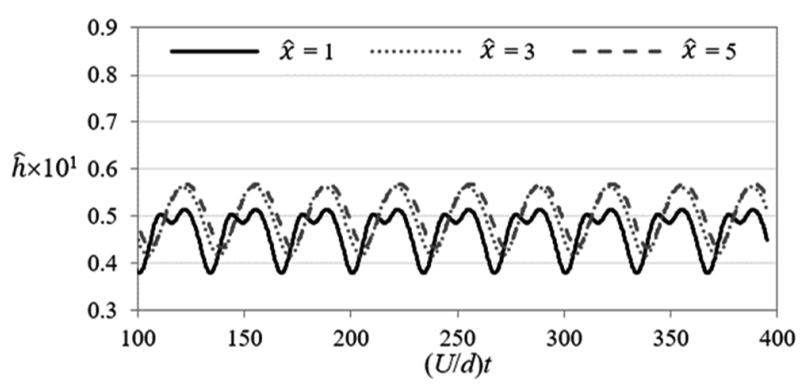

(a)

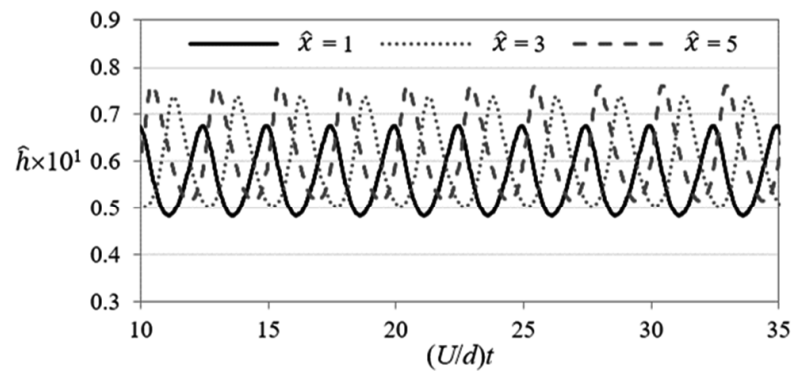

(b)

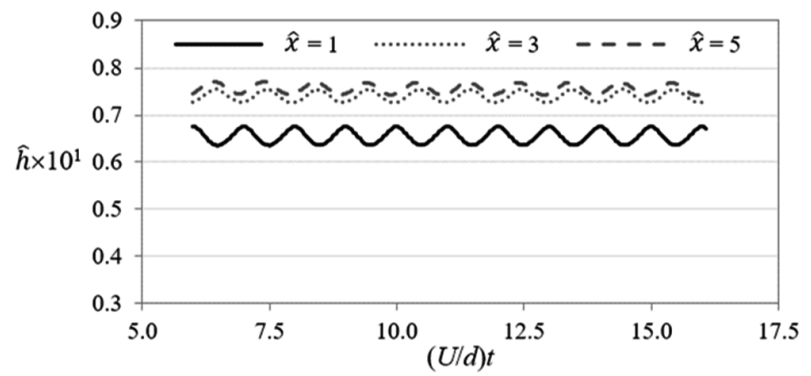

(c)

Fig. 9. Time-traces of the coating thickness for a) Case 1: $\left.d f_{\text {pos }} / U=0.030, U=1.0 \mathrm{~m} / \mathrm{s}, a_{\text {pos }} / d=1.0, f_{\text {pos }}=30 \mathrm{~Hz} ; \mathrm{b}\right)$ Case 2: $d f_{\text {pos }} / U=0.40, U=1.5 \mathrm{~m} / \mathrm{s}, a_{\text {pos }} / d=0.5, f_{\text {pos }}=$ $600 \mathrm{~Hz}$; and c) Case 3: $d f_{\text {pos }} / U=1.0, U=2.0 \mathrm{~m} / \mathrm{s}, a_{\text {pos }} / d=$ $0.4, f_{\text {pos }}=2000 \mathrm{~Hz}$.

at $U=2 \mathrm{~m} / \mathrm{s}$ the bandwidth is the approximate range $0-1$ $\mathrm{kHz}$ compared to roughly $0-2 \mathrm{kHz}$ for $U=4 \mathrm{~m} / \mathrm{s}$. This $U$-bandwidth characteristic is of particular significance to operation at higher strip speeds on CGL given frequency spectra of impinging planar jets typically include significant energy content at frequencies as low as $1 \mathrm{kHz}$.

Consider, for example, an assumed scenario for a jet lip gap of $d=1 \mathrm{~mm}$ where perturbations are present in the wip- 
Table 3. Average thickness reduction ratio $\hat{h}_{\text {mean }}$ at $\hat{x}=5$ of the three example cases.

\begin{tabular}{ccc}
\hline Case & Strip speed, $U[\mathrm{~m} / \mathrm{s}]$ & $\hat{h}_{\text {mean }} \times 10^{1}$ \\
\hline 1 & 1.0 & 0.51 \\
2 & 1.5 & 0.61 \\
3 & 2.0 & 0.76 \\
\hline
\end{tabular}

ing jet flow at $f=1 \mathrm{kHz}$ (where the notation $f$ is adopted herein to represent a particular frequency component of the typically broadband oscillations of impinging planar jets). Under these circumstances operating line speeds of up to $U \sim 2 \mathrm{~m} / \mathrm{s}$ are relatively insensitive to the perturbations at this frequency (see Fig. 8) and, accordingly, the amplitude of the coating ripple generated will be quite small. However, if the strip speed is further increased to, say, $3 \mathrm{~m} / \mathrm{s}$ the coating becomes highly sensitive to the $1 \mathrm{kHz}$ jet perturbations and a coating ripple of significant amplitude will result. Note that, as Fig. 8 shows, the worst-case scenario for $U=$ $3 \mathrm{~m} / \mathrm{s}$ when using a jet lip gap of $d=1 \mathrm{~mm}$ is perturbations at $f=0.66 \mathrm{kHz}$ (which corresponds to $d f / U=0.22$ ).

Finally, in Fig. 9 the localised coating thickness variation over time as a consequence of the unsteady forcing is presented for the three previously discussed examples: Cases 1, $2 \& 3$. In each sub-plot of Fig. 9 the thickness over time is shown at three selected downstream strip locations $\hat{x}=1,3$ $\& 5$. Evidently the average value of the thickness reduction ratio $\hat{h}$ is different between the three example cases. This outcome is as expected, however, given the strip velocity is different in each case (see Table 3 ) whilst the magnitude and spread of the pressure and shear stress profiles was identical.

Note the accordance in Fig. 9 between the peak-to-peak ranges-relative to the respective average thicknesses (see $\hat{h}_{\text {mean }}$ values in Table 3)-of the curves for strip location $\hat{x}=$ 5 and the corresponding $r_{p o s}$ (Eq. (14)) and $r_{p o s-m o d}$ (Eq. (16)) values of Figs. 6 and 7, respectively.

\section{Conclusion}

A numerical model has been developed and used to simulate the response of the coating film of the jet-wiping problem when subjected to unsteady pressure and shear stress acting on the surface of the coating; specifically, under idealised conditions of vertical oscillation in the location of both the pressure and shear stress profiles occurring in a sinusoidal manner at prescribed amplitude and frequency. Film properties typical of liquid metallic coatings employed in industrial continuous galvanising lines are assumed and the breadth and magnitude of the pressure and shear stress profiles accord to time-averaged results observed for nondimensional jet-strip offset $H / d \sim 8$ at jet Reynolds number of $\sim 7 \times 10^{3}$.

When subjected to the aforementioned unsteady pressure and shear stress, the amplitude of the ripple (relative to the average thickness of the coating) generated on the coating surface is found to exhibit a dependency on the frequency with which the pressure and shear stress oscillate. At above unity values of the nondimensional frequency $d f_{\text {pos }} / U$ the coating exhibits very little variation in thickness (i.e. small values of the $r_{\text {pos-mod }}$ parameter). However, as nondimensional frequency is reduced to lower values the magnitude of thickness variation is found to monotonically increase up to the point where a peak occurs at nondimensional frequency of $d f_{p o s} / U \sim 0.22$. At this operating point the amplitude of the coating ripple-relative to the average thickness of the coating-is greatest. That is, the coating is indicated by the simulation results to be most sensitive to perturbations in the jet wiping when the frequency of the perturbations corresponds to the operating point $d f_{\text {pos }} / U \sim$ 0.22 . With further reduction in the nondimensional frequency the ripple amplitude monotonically decreases from the value at the peak. However, the ripple amplitude in this low $d f_{\text {pos }} / U$ region (i.e. $d f_{\text {pos }} / U<\sim 0.1$ ) is approximately an order-of-magnitude greater than that of the high $d f_{p o s} / U$ region (i.e. $d f_{\text {pos }} / U>\sim 1$ ).

Overall, the outcomes suggest that the consistency in the thickness of the coating film produced on continuous galvanising lines may be expected to be most highly affected when, considered together with the size of the jet lip gap $d$ and the speed of the strip $U$, a frequency component $f$ of one or more of the modes of the coherent structures comprising the unsteady impinging jet flow corresponds to nondimensional frequency at or near $d f / U=0.22$. In order to achieve smooth coatings of consistent coating weight (i.e. consistent thickness) the most desirable operating range is the high nondimensional frequency region $d f / U>1$.

\section{Acknowledgements}

Funding from the Australian Research Council Industrial Transformation Research Hubs Scheme (Project Number IH130100017) is gratefully acknowledged.

\section{REFERENCES}

1) A. D. Weir, D. H. Wood and P. Bradshaw: J. Fluid Mech., 107 (1981), 237.

2) L. W. B. Browne, R. A. Antonia and A. J. Chambers: J. Fluid Mech., 149 (1984), 355.

3) L. O. Merkine and J. T. C. Liu: J. Fluid Mech., 70 (1975), 353.

4) R. A. Antonia, L. W. B. Browne, S. Rajagopalan and A. J. Chambers: J. Fluid Mech., 134 (1983), 49

5) H. Sato: J. Fluid Mech., 7 (1960), 53.

6) F. O. Thomas and V. W. Goldschmidt: J. Fluid Mech., 163 (1986), 227.

7) I. Namer and M. V. Ötügen: Exp. Fluids, 6 (1988), 387.

8) Y. Guo and D. H. Wood: Exp. Therm. Fluid Sci., 25 (2002), 605

9) D. Arthurs and S. Ziada: Exp. Fluids, 55 (2014), 1.

10) E. Gutmark, M. Wolfshtein and I. Wygnanski: J. Fluid Mech., 88 (1978), 737

11) C. Pfeiler, W. Eß1, G. Reiss, C. K. Riener, G. Angeli and A. Kharicha: Steel Res. Int., 88 (2017), 1600507.

12) W. Eß1, C. Pfeiler, G. Reiss, W. Ecker, C. K. Riener and G. Angeli: Steel Res. Int., 89 (2018), 1700362.

13) D. Arthurs and S. Ziada: J. Fluid. Struct., 27 (2011), 105.

14) H. H. Assoum, A. Sakout, K. Abed-Meraïm, A. Alia, M. E. Hassan and J. Vetel: Proc. 9th European Conf. on Noise Control, CAS, Prague, (2012), 801.

15) M. Mendez, K. Myrillas, A. Gosset and J.-M. Buchlin: European Coating Symp. (ECS15) Extended Abstracts, Technische Universiteit Eindhoven, Eindhoven, (2015), 22.

16) C. Mugrauer, M. Mataln, A. Kharicha, C. Riener and G. Angeli: Int. Conf. on Zinc and Zinc Alloy Coated Steel Sheet (Galvatech 2015), AIST, Warrendale, PA, (2015), 682.

17) G. C. Hocking, W. L. Sweatman and A. D. Fitt: J. Eng. Math., 70 (2011), 297.

18) C. S. Yih: Phys. Fluids, 6 (1963), 321

19) C. H. Ellen and C. V. Tu: Phys. Fluids, 28 (1985), 1202.

20) E. A. Elsaadawy, G. S. Hanumanth, A. K. S. Balthazaar, J. R. McDermid, A. N. Hrymak and J. F. Forbes: Metall. Mater. Trans. B, 38 (2007), 413.

21) C. V. Tu and D. H. Wood: Exp. Therm. Fluid Sci., 13 (1996), 364.

22) A. D. Johnstone, B. Kosasih, L. Q. Phan, A. Dixon and W. Renshaw: Proc. Int. Conf. on Zinc and Zinc Alloy Coated Steel Sheet (Galvatech 2017), ISIJ, Tokyo, (2017), 314. 